\title{
ClearAlert: a fieldable sensor for rapid autonomous detection of solid and aerosol fentanils
}

Piercen Oliver, Madison Myers, Amanda Code

Piercen Oliver, Madison Myers, Amanda Code, "ClearAlert: a fieldable sensor for rapid autonomous detection of solid and aerosol fentanils," Proc. SPIE 11749, Chemical, Biological, Radiological, Nuclear, and Explosives (CBRNE) Sensing XXII, 1174903 (12 April 2021); doi: 10.1117/12.2588222 


\section{ClearAlert a fieldable sensor for rapid autonomous detection of solid and aerosol fentanils (Withdrawal Notice)}

Piercen Oliver, Madison Myers, and Amanda Code

Clear Scientific, Inc. (United States)

Proceedings Volume 11749, Chemic al, Biologic al, Radiological, Nuc lear, and Explosives (CBRNE) Sensing XXIl; 1174903 (2021) https:// doi.org/10.1117/12.2588222

Event SPIE Defense + Commerc ial Sensing Digital Forum, 2021, O nline Only, United States

Online Publication Date: 13 April 2021

Withdrawn from Publication: 4 May 2021

Publisher's Note: This pa per, orig ina lly published on 13 April 2021, was withdrawn per a uthor request. 